\title{
Homocystinuria with Lower Gastrointestinal Bleeding: First Case Report
}

\author{
Mohammad Al Humaidan ${ }^{a}$ Ibrahim Al Sharkawy ${ }^{a} \quad$ Abdullah Al Sanae $^{b}$ \\ Fawaz Al Refaee ${ }^{c}$ \\ ${ }^{a}$ Department of Pediatrics, AlSabah Hospital, Kuwait City, ${ }^{b}$ Chest Hospital, Kuwait City, and ${ }^{\mathrm{C}}$ AlAdan Hospital, \\ AlFahaheel, Kuwait
}

\section{Key Words}

Homocystinuria $\cdot$ Abdominal pain $\cdot$ Child health $\cdot$ Lower gastrointestinal bleeding $\cdot$ Thrombosis

\section{Abstract}

Objective: To report a rare complication of homocystinuria in a child and highlight the association of homocystinuria with lower gastrointestinal bleeding and intestinal thrombosis. Clinical Presentation and Intervention: A 7-year-old boy with homocystinuria and poor compliance with treatment presented with abdominal pain and bloody stools. Doppler ultrasound showed superior mesenteric and middle colic vein thrombosis. Laparotomy demonstrated ischemic small bowel necessitating resection. The patient improved clinically following resection and the initiation of the anticoagulation with homocystinuria treatment and was discharged home. Conclusion: This case showed the need to sustain a high index of suspicion of thromboembolism in a patient with homocystinuria and lower gastrointestinal bleeding and the importance of compliance with treatment to avoid vascular complications.

Copyright $\odot 2012$ S. Karger AG, Basel

\section{Introduction}

Homocystinuria is a rare genetically determined inborn error of metabolism of methionine and prevalence has been estimated to be 1 in 344,000 [1]. This autosomal recessive inherited disorder is characterized by abnormality in transsulfuration pathway of methionine metabolism secondary to cystathionine B synthase deficiency. Biochemically, this disorder is associated with accumulation of methionine, homocysteine and their $\mathrm{S}$-adenosyl derivatives in the tissue with lack of cystathionine and a low level of cysteine. Clinically, affected patients present with eye, skeleton, central nervous system and, most importantly, vascular system abnormalities $[2,3]$.

The association between homocystinuria and the vascular complications was first reported in 1976 [4], and since then several studies have confirmed this association $[5,6]$. Thromboembolic events affect arteries and veins of all parts of the body, representing a major cause of morbidity and mortality. The most commonly reported events are cerebrovascular accident, pulmonary embolism, carotid, and renal arteries thrombosis 
[7]. We present a rare association between homocystinuria and gastrointestinal (GI) bleeding in a 7-year-old boy.

\section{Case Report}

A 7-year-old boy known to have homocystinuria since the age of 2 years and 10 months presented to the pediatric emergency department with abdominal pain and bloody stools. The child was born full term by cesarean section delivery with a birth weight of $2.7 \mathrm{~kg}$. He was born to healthy consanguineous parents and has 5 brothers and 3 sisters. The child was growing normally except for delayed speech development. At the age of 2 years he started to have recurrent focal seizure alternating from one side to the other and associated with a disturbed level of consciousness. Electroencephalogram (EEG) showed focus of sharp waves on the right centroparietal and temporal regions. Computed tomography of the head was performed and reported as being normal. The patient was started on different antiepileptic medications which partially controlled the seizures.

Ten months later, the patient developed right-sided hemiparesis. Computed tomography of the head showed a hypodense area on the left thalamic region. Magnetic resonance imaging and magnetic resonance venography of the brain showed left thalamic hemorrhagic infarction and thrombosis of both the internal cerebral vein and vein of Galen. Following the magnetic resonance venography, the child developed deep vein thrombosis extending from the right femoral vein up to the common iliac vein. Anticoagulation therapy (heparin followed by warfarin) was started along with the antiepileptic medication. Further investigations revealed positive urine and blood for homocystinuria. Eye examination demonstrated bilateral downward lens dislocation. Sibling screenings were positive for homocystinuria for 1 brother and 1 sister. Vitamin $\mathrm{B}_{6}$, folic acid and betaine were added to the treatment and 8 weeks later warfarin was discontinued and dipyridamole was added.

The child improved gradually and showed signs of complete recovery. He started to walk and talk normally 8 weeks following the event. However, the child was not compliant with the treatment and did not attend the outpatient follow-up regularly. Despite the lack of compliance, the child became seizure-free and antiepileptic medication was stopped at the age of $5 \frac{1}{2} / 2$ years.

At the age of 7 years he had symptoms of upper respiratory tract infection with vomiting. An oral antibiotic was prescribed by his family physician. Several days later he showed up in the emergency room with crampy abdominal pain and bloody stools. Physical examination showed normal vital signs with no signs of dehydration or infections. He was admitted to the hospital for further investigations. During his stay, the child continued to have progressively worsening abdominal pain with lower GI bleeding; he was therefore transferred to the surgical side. Doppler ultrasound of the abdomen showed superior mesenteric vein and middle colic vein thrombosis. The patient underwent an emergency exploratory laparotomy which demonstrated a 120$\mathrm{cm}$ ischemic black small bowel approximately $50 \mathrm{~cm}$ distal to the duodenojejunal junction. Resection with end-to-end anastomosis was performed and anticoagulant therapy was commenced after the procedure. A few days later the patient started to tolerate oral fluid and vitamin $\mathrm{B}_{6}$, betaine, folic acid and methionine restriction were added. He was discharged in a good general condition. Homocysteine and methionine levels on admission were 24 and $739 \mu \mathrm{mol} / \mathrm{l}$, respectively, while on discharge corresponding values were 11 and $265 \mu \mathrm{mol} / \mathrm{l}$ (normal range: methionine 10-40 $\mu \mathrm{mol} / \mathrm{l}$, homocysteine not detected). The discharge medication included vitamin $\mathrm{B}_{6}$, betaine, folic acid, and warfarin. The child and the family were advised to be compliant with treatment and clinic appointments. With subsequent visits he was noted to be compliant with the medications and the follow-up as well as his affected siblings.

\section{Discussion}

Homocystinuria is the second most common treatable aminoacidopathy [8]. Marked accumulation of homocysteine is frequently associated with multisystem involvements. Vascular complication continues to be a major cause of morbidity and mortality. It has been shown that before the age of 30 years, more than half of the affected patients will suffer at least one thromboembolic event [2]. Although theoretically the thromboembolic event occurs in any vein or artery, the most commonly reported sites are the central nervous system, lungs, carotids, and renal arteries [7]. Our patient had homocystinuria associated with lower GI bleeding. The patient reported here is a 7-year-old boy known to have homocystinuria with poor compliance who presented with abdominal pain and lower GI bleeding with no signs of infection or dehydration. Despite admission to the hospital and supportive care, he continued to show progressively worsening symptoms. Eventually he was found to have superior mesenteric vein thrombosis and middle colic vein thrombosis. A thromboembolic event can occur at any age in a patient with homocystinuria. The mechanism by which homocysteine causes vascular injury is not fully understood; however, there is evidence in the literature $[9,10]$ suggesting that a high concentration of homocysteine induces vascular endothelial injury. This is probably explained by the generation of hydrogen peroxide and reactive oxygen species due to oxidation of homocysteine to homocystine which may inactivate endothelium-derived nitric oxide and thrombomodulin and result in platelet aggregation, vasoconstriction, and impaired anticoagulation [10].

This case revealed that thrombosis of the intestinal vessels is possible and should be highly suspected in a patient with homocystinuria and lower GI bleeding. Lack of compliance may have played a role in developing disease complications as in our case, probably because the child 
was not compliant with his treatment and did not attend the outpatient follow-up regularly. To avoid potentially life-threatening vascular complications, compliance with treatment such as vitamin $\mathrm{B}_{6}$, betaine, folic acid and methionine restriction should be emphasized to patients with homocystinuria.

\section{Conclusion}

This case showed that physicians should sustain a high index of suspicion of thromboembolic events in patients presenting with signs and symptoms of homocystinuria and lower GI bleeding as well as the importance of treatment compliance to avoid developing vascular complications.

\section{References}

1 Mudd SH, Levy HL, Skovby F: Disorders of transsulfuration; in Scriver CR, Beaudet AL, Sly WS, Valle D (eds): The Metabolic and Molecular Bases of Inherited Disease, ed 7. New York, McGraw-Hill, 1995, pp 12791327.

2 Mudd SH, Skovby F, Levy HL, Pettigrew KD, Wilcken B, Pyeritz RE, Andria G, Boers GH, Bromberg IL, Cerone R, Fowler B, Grobe H, Schemidt H, Schweitzer L: The natural history of homocystinuria due to cystathionine $\beta$-synthase deficiency. Am J Hum Genet 1985;37:1-31.
-3 De Franchis R, Sperandeo MP, Sebastio G, Andria G: Clinical aspects of cystathionine $\beta$-synthase deficiency: how wide is the spectrum? The Italian Collaborative Study Group on Homocystinuria. Eur J Pediatr 1998;157(suppl 2):S67-S70.

4 Wilcken DE, Wilcken B: The pathogenesis of coronary artery disease: a possible role for methionine metabolism. J Clin Invest 1976; 57:1079-1082.

5 Hankey GJ, Eikelboom JW: Homocysteine and vascular disease. Lancet 1999;354:407413.

6 Mojiminiyi OA, Marouf R, Al Shayeb AR, Qurton M, Abdella NA, Al Wazzan H, Al Humood S, Samad MA, El-Muzaini H: Determinants and associations of homocysteine and prothrombotic risk factors in $\mathrm{Ku}$ waiti patients with cerebrovascular accident. Med Princ Pract 2008;17:136-142.
7 Generoso A, Fowler B, Sebastio G: Disorders of sulfur amino acid metabolism; in Fernandes J, Saudubray J, Berghe G, Walter J (eds): Inborn Metabolic Diseases: Diagnosis and Treatment, ed 4. Berlin, Springer, 2006, pp 273-281.

$>$ Yap S: Classical homocystinuria: vascular risk and its prevention. J Inherit Metab Dis 2003;26:259-265.

9 Cattaneo M: Hyperhomocysteinemia and thrombosis. Lipids 2001;36:531-526.

10 Lentz SR: Homocysteine and vascular dysfunction. Life Sci 1997;61:1205-1215. 